\title{
Analysis of a Bidirectional Coupled-Inductor Ćuk Converter Operating in Sliding Mode
}

\author{
Luis Martínez-Salamero, Senior Member, IEEE, Javier Calvente, Roberto Giral, Alberto Poveda, and Enric Fossas
}

\begin{abstract}
Analytic models for a bidirectional coupled-inductor Ćuk converter operating in sliding mode are described. Using a linear combination of the converter four state variable errors as a general switching surface, the expression for the equivalent control is derived and the coordinates of the equilibrium point are obtained. Particular cases of the general switching surface are subsequently analyzed in detail: 1) surfaces for ideal line regulation, 2) surfaces for ideal load regulation, and 3) surfaces for hysteretic current control. Simulation results verifying the analytical predictions are presented.
\end{abstract}

Index Terms - DC-to-DC switching converters, sliding mode, variable structure systems.

\section{INTRODUCTION}

$\mathbf{C}$ OUPLED-INDUCTOR Ćuk converters have been extensively used in the design of high efficiency minimum weight power processing units with extremely low conducted noise requirements [1]-[3]. Due to the significant properties of the Ćuk converter, a considerable amount of research has been devoted to the modeling, analysis, and applications of that structure [4]-[8].

However, in spite of the converter topological simplicity, the dynamic analysis constitutes a more involved problem. The controller design following the state-space averaging method [4], based on the use of a pulse width modulator (PWM) and a linear low-frequency characterization of the converter, is rather difficult in the two conduction modes. In the continuous conduction mode, the dynamic response exhibits a four order nonminimum phase behavior which requires bandwidth limitations to guarantee the regulator closed-loop stability. This requirement is accomplished by using a big capacitor to transfer the energy between input and output

Manuscript received October 10, 1996; revised March 6, 1997. This work was supported in part by the Ministerio de Educación y Ciencia (Spain) under Grant CICYT-TAP94-0552-C03-03. This paper was recommended by Associate Editor A. Ioinovici.

L. Martínez-Salamero, J. Calvente, and R. Giral are with the Escola Tècnica Superior d'Enginyeria, Departament d'Enginyeria Electrònica, Universitat Rovira i Virgili, Carretera de Salou s/n, 43006 Tarragona, Spain (e-mail: rgiral@etse.urv.es).

A. Poveda is with the Escola Tècnica Superior d'Enginyers de Telecomunicació, Departament d'Enginyeria Electrònica, Universidad Politécnica de Cataluña, Mòdul C4, Campus Nord, c/ Gran Capità s/n, 08034 Barcelona, Spain.

E. Fossas is with the Departament de Matemàtica Aplicada i Telemàtica, Universidad Politécnica de Cataluña, Mòdul C3, Campus Nord, c/ Gran Capità s/n, 08034 Barcelona, Spain.

Publisher Item Identifier S 1057-7122(98)02112-6. stages. Moreover, the analysis of the dynamic response is considerably complex in the discontinuous conduction mode, where the instant in which the switch current becomes zero depends on the converter state and not on its duty cycle [9].

In order to avoid the discontinuous conduction mode and, at the same time, to allow the existence of light load levels, the bidirectional switch was introduced [10], this having led to a bilinear description of the converter [16]. However, the corresponding small signal model based on the state-space averaging method cannot predict the regulator behavior when a large-signal perturbation appears.

These drawbacks have prompted some authors to explore nonlinear techniques based on the application of variable structure theory [11], [12] and the associated sliding regimes. The sliding-mode control is a time-domain technique suitable for large-signal applications exhibiting low sensitivity to external perturbations. Sliding mode analysis of the coupledinductor Ćuk converter has been tangentially treated in the work of Sira-Ramirez on sliding motions in switching power converters [17]. The sliding surfaces considered are of the form $x_{j}=K$, for some state variable that is desired to be regulated at the level $K$. The restriction to this type of surface has disadvantages. Namely, only the sliding surface representing constant input current leads to an stable equilibrium point, but it does not provide output voltage regulation. Papers [18]-[21] deal specifically with the Cuk converter operating in sliding mode. The work reported in [18] assumes that the capacitor for energy transference is considerably bigger than the output capacitor in order to decouple input and output stages. With this parametric constraint, the coupled-inductor Ćuk converter can be controlled like a buck switching cell [14], [15], but the input current exhibits an uncontrollable dynamic behavior. In another direction, [19] has considered time-varying surfaces to attenuate perturbations effects caused by input voltage or load variations. A relative insensitivity of the output voltage to such variations has been demonstrated with simulations. However, the implementation of the control algorithm is complex and requires accurate parameter and state information. The development of sliding mode control laws for a Ćuk converter without magnetic coupling is the subject of the work of Malesani et al. reported in [20]. The sliding surface considered in [20] is a linear combination of the four state variable errors resulting in simple control implementation, minimum size of energy transfer capacitor, and excellent static and dynamic performances. However, there is a lack of analytic investigation on the influence of 


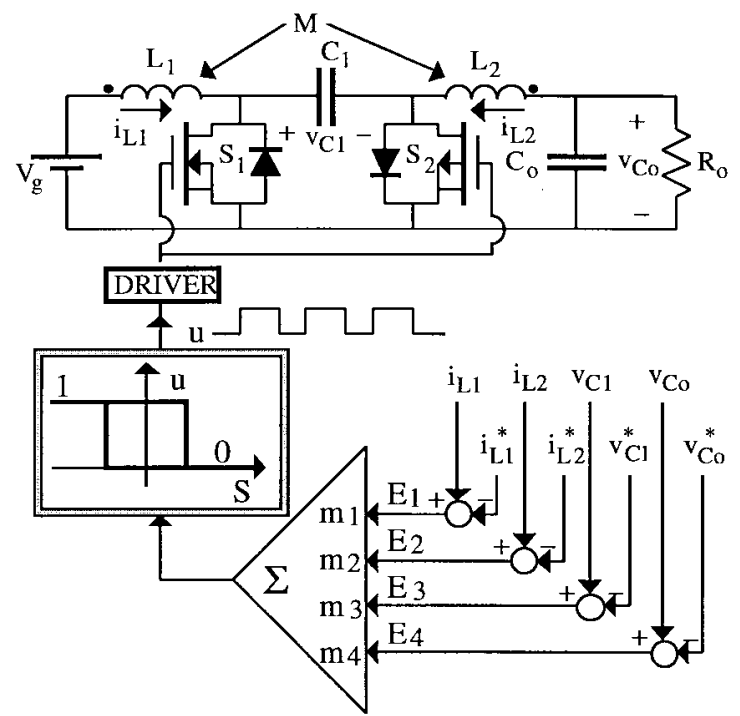

Fig. 1. Block diagram of a sliding-mode controlled bidirectional coupled-inductor Ćuk switching regulator.

the surface coefficients on the regulator behavior, these coefficients being eventually obtained through numerical analysis. In a more recent paper [21], a sliding mode control strategy has been proposed for a third-order Ćuk converter. Analytic expressions are given for the equilibrium points corresponding to switching surfaces that are linear combinations of up to three state variables. The corresponding experimental results show a relative insensitivity of the output voltage with respect to input voltage variations. However, the absence of the output filtering capacitor results in a significant amount of ripple in the output voltage which makes the third-order Cuk converter inappropriate for practical purposes.

The aim of this paper is to develop analytic models for the dynamic behavior of a bidirectional coupled-inductor Ćuk converter operating in sliding mode. The approach is based on the equivalent control method [17] and uses a linear combination of the converter state variable errors as a general switching surface as shown in Section II. Section III is devoted to the study of different particular cases of the general sliding surface such as surfaces for ideal line regulation, ideal load regulation, and hysteretic current control. Section IV contains conclusions and some suggestions for further research.

\section{SLIDING DOMAIN}

Fig. 1 shows the block diagram of a sliding-mode controlled bidirectional coupled-inductor Ćuk switching regulator. Transistors and diodes are assumed to be ideal, and no parasitics or storage time modulation effects have been considered. In addition, losses in the passive components have been neglected.

Due to the bidirectional current capability of the switch, the discontinuous conduction mode does not take place, and therefore the converter has only one structural change during a switching period. Considering $u=1$ during the switch $S_{1}$ conduction subinterval and $u=0$ during the switch $S_{2}$ conduction subinterval, the converter dynamics can be described as follows:

$$
\begin{aligned}
\frac{d i_{L 1}}{d t} & =-\frac{v_{C 1}}{L_{1}}(1-u)+\frac{V_{g}}{L_{1}}-\frac{M}{L_{1}} \frac{d i_{L 2}}{d t} \\
\frac{d i_{L 2}}{d t} & =\frac{v_{C 1}}{L_{2}} u+\frac{v_{C o}}{L_{2}}-\frac{M}{L_{2}} \frac{d i_{L 1}}{d t} \\
\frac{d v_{C 1}}{d t} & =-\frac{i_{L 2}}{C_{1}} u+\frac{i_{L 1}}{C_{1}}(1-u) \\
\frac{d v_{C o}}{d t} & =-\frac{i_{L 2}}{C_{o}}-\frac{v_{C o}}{R_{o} C_{o}} .
\end{aligned}
$$

Defining the normalized variables $x_{1}=\sqrt{L_{1}} i_{1}, x_{2}=$ $\sqrt{L_{2}} i_{2}, x_{3}=\sqrt{C_{1}} v_{C 1}, x_{4}=\sqrt{C_{o}} v_{C o}$, (1)-(4) become

$$
\begin{aligned}
& \dot{x}_{1}=-\frac{\omega_{1} x_{3}}{1-k^{2}}(1-u)+\frac{e}{1-k^{2}}-\frac{k \omega_{21} x_{3}}{1-k^{2}} u-\frac{k \omega_{2} x_{4}}{1-k^{2}} \\
& \dot{x}_{2}=\frac{\omega_{21} x_{3}}{1-k^{2}} u+\frac{\omega_{2} x_{4}}{1-k^{2}}+\frac{k \omega_{1} x_{3}}{1-k^{2}}(1-u)-\frac{k e}{1-k^{2}} \\
& \dot{x}_{3}=-\omega_{21} x_{2} u+\omega_{1} x_{1}(1-u) \\
& \dot{x}_{4}=-\omega_{2} x_{2}-\omega_{R} x_{4}
\end{aligned}
$$

where

$$
\begin{aligned}
\omega_{1} & =\frac{1}{\sqrt{L_{1} C_{1}}}, \omega_{2}=\frac{1}{\sqrt{L_{2} C_{o}}}, \\
\omega_{21} & =\frac{1}{\sqrt{L_{2} C_{1}}}, \omega_{R}=\frac{1}{R_{o} C_{o}}, \\
e & =\frac{V_{g}}{\sqrt{L_{1}}}, k=\frac{M}{\sqrt{L_{1} L_{2}}} \quad(-1 \leq k \leq 1) .
\end{aligned}
$$

Writing (5)-(8) in matrix form results in

$$
\begin{aligned}
{\left[\begin{array}{c}
\dot{x}_{1} \\
\dot{x}_{2} \\
\dot{x}_{3} \\
\dot{x}_{4}
\end{array}\right]=} & {\left[\begin{array}{cccc}
0 & 0 & \frac{-\omega_{1}}{1-k^{2}} & \frac{-k \omega_{2}}{1-k^{2}} \\
0 & 0 & \frac{k \omega_{1}}{1-k^{2}} & \frac{\omega_{2}}{1-k^{2}} \\
\omega_{1} & 0 & 0 & 0 \\
0 & -\omega_{2} & 0 & -\omega_{R}
\end{array}\right]\left[\begin{array}{l}
x_{1} \\
x_{2} \\
x_{3} \\
x_{4}
\end{array}\right] } \\
& +u\left[\begin{array}{cccc}
0 & 0 & \frac{\omega_{1}-k \omega_{21}}{1-k^{2}} & 0 \\
0 & 0 & \frac{\omega_{21}-k \omega_{1}}{1-k^{2}} & 0 \\
-\omega_{1} & -\omega_{21} & 0 & 0 \\
0 & 0 & 0
\end{array}\right]\left[\begin{array}{l}
x_{1} \\
x_{2} \\
x_{3} \\
x_{4}
\end{array}\right] \\
& +\left[\begin{array}{c}
\frac{e}{1-k^{2}} \\
-\frac{k e}{1-k^{2}} \\
0 \\
0
\end{array}\right] .
\end{aligned}
$$

Consider now as sliding surface $S(x)$ a linear combination of state variable errors $E_{i}$

$$
S(x)=m_{1} E_{1}+m_{2} E_{2}+m_{3} E_{3}+m_{4} E_{4}
$$

where $E_{1}=i_{L 1}-i_{L 1}^{*}, E_{2}=i_{L 2}-i_{L 2}^{*}, E_{3}=v_{C 1}-v_{C 1}^{*}$, $E_{4}=v_{C o}-v_{C o}^{*}$, and $\left(i_{L 1}^{*}, i_{L 2}^{*}, v_{C 1}^{*}, v_{C o}^{*}\right)$ are the desired values of the equilibrium point coordinates. 
In terms of the normalized variables, (10) becomes

$$
S(x)=\alpha x_{1}+\beta x_{2}+\gamma x_{3}+\delta x_{4}-\theta .
$$

On the average, the system motion takes place on the surface $S(x)=0$, whose corresponding gradient $d S$ will be given by

$$
d S=(\alpha, \beta, \gamma, \delta)
$$

Equation (9) can also be expressed as follows:

$$
\dot{x}=(A x+D)+(B x+C) u
$$

where

$$
\begin{aligned}
& A=\left[\begin{array}{cccc}
0 & 0 & \frac{-\omega_{1}}{1-k^{2}} & \frac{-k \omega_{2}}{1-k^{2}} \\
0 & 0 & \frac{k \omega_{1}}{1-k^{2}} & \frac{\omega_{2}}{1-k^{2}} \\
\omega_{1} & 0 & 0 & 0 \\
0 & -\omega_{2} & 0 & -\omega_{R}
\end{array}\right] \quad D=\left[\begin{array}{c}
\frac{e}{1-k^{2}} \\
-\frac{k e}{1-k^{2}} \\
0 \\
0
\end{array}\right] \\
& B=\left[\begin{array}{cccc}
0 & 0 & \frac{\omega_{1}-k \omega_{21}}{1-k^{2}} & 0 \\
0 & 0 & \frac{\omega_{21}-k \omega_{1}}{1-k^{2}} & 0 \\
-\omega_{1} & -\omega_{21} & 0 & 0 \\
0 & 0 & 0 & 0
\end{array}\right]
\end{aligned}
$$

$C=\emptyset \quad$ (null matrix)

A necessary condition for the existence of a sliding motion in $S(x)$ is given by the transversality condition [17]

$$
\langle d S, B x+C\rangle \neq 0
$$

where the notation $\langle a, b\rangle$ denotes the scalar product of $a$ and $b$.

From (12)-(14), we derive

$$
\begin{aligned}
& \alpha \frac{\omega_{1}-k \omega_{21}}{1-k^{2}} x_{3}+\beta \frac{\omega_{21}-k \omega_{1}}{1-k^{2}} x_{3}-\gamma \omega_{1} x_{1} \\
& -\gamma \omega_{21} x_{2} \neq 0 \text {. }
\end{aligned}
$$

Hence, surfaces of the form $\delta x_{4}=\theta$ are disregarded since they cannot lead the converter to a sliding regime.

In the method of equivalent control, the discrete function $u$ is substituted by a continuous function $u_{\mathrm{eq}}(x)$ which can take all the values between 0 and $1 . U_{\text {eq }}(x)$ is the equivalent control and represents the control law that describes the behavior of the system restricted to the switching surface. The expression of $u_{\text {eq }}(x)$ [17] is given by

$$
u_{\mathrm{eq}}(x)=-\frac{\langle d S, A x+D\rangle}{\langle d S, B x+C\rangle} .
$$

From (12)-(16), we obtain (17), shown at the bottom of the page, which is bounded by the minimum and maximum values of $u$

$$
0<u_{\text {eq }}<1
$$

thus providing the sliding domain.

Substituting $u$ by $u_{\mathrm{eq}}(x)$ in (5)-(8) results in the following ideal sliding dynamics:

$$
\begin{aligned}
& \dot{x}_{1}=-\frac{\omega_{1} x_{3}}{1-k^{2}}\left(1-u_{\mathrm{eq}}\right)+\frac{e}{1-k^{2}}-\frac{k \omega_{21} x_{3}}{1-k^{2}} u_{\mathrm{eq}}-\frac{k \omega_{2} x_{4}}{1-k^{2}} \\
& \dot{x}_{2}=\frac{\omega_{21} x_{3}}{1-k^{2}} u_{\mathrm{eq}}+\frac{\omega_{2} x_{4}}{1-k^{2}}+\frac{k \omega_{1} x_{3}}{1-k^{2}}\left(1-u_{\mathrm{eq}}\right)-\frac{k e}{1-k^{2}} \\
& \dot{x}_{3}=-\omega_{21} x_{2} u_{\mathrm{eq}}+\omega_{1} x_{1}\left(1-u_{\mathrm{eq}}\right) \\
& \dot{x}_{4}=-\omega_{2} x_{2}-\omega_{R} x_{4}
\end{aligned}
$$

where $u_{\text {eq }}(x)$ is given by (17).

The equilibrium point of (19)-(22) results from the intersection of the curve of equilibrium points and the discontinuity surface. Namely,

$$
\begin{aligned}
x_{1}^{*} & =\frac{\omega_{R}}{e}\left(x_{4}^{*}\right)^{2} \\
x_{2}^{*} & =-\frac{\omega_{R}}{\omega_{2}} x_{4}^{*} \\
x_{3}^{*} & =\frac{e}{\omega_{1}}-x_{4}^{*} \frac{\omega_{2}}{\omega_{21}} \\
\theta & =\alpha x_{1}^{*}+\beta x_{2}^{*}+\gamma x_{3}^{*}+\delta x_{4}^{*} .
\end{aligned}
$$

From (23)-(26) we derive

$$
\frac{\alpha \omega_{R}}{e} x_{4}^{* 2}+\left(\delta-\frac{\beta \omega_{R}}{\omega_{2}}-\frac{\gamma \omega_{2}}{\omega_{21}}\right) x_{4}^{*}+\frac{\gamma e}{\omega_{1}}-\theta=0
$$

and therefore $x_{4}^{*}$ will be given by (28), shown at the bottom of the page, or

$$
\begin{gathered}
x_{4}^{*}=\frac{\theta-\frac{\gamma e}{\omega_{1}}}{\delta-\frac{\beta \omega_{R}}{\omega_{2}}-\frac{\gamma \omega_{2}}{\omega_{21}}} \quad \text { if } \alpha=0 \text { and } \\
\delta-\frac{\beta \omega_{R}}{\omega_{2}}-\frac{\gamma \omega_{2}}{\omega_{21}} \neq 0 .
\end{gathered}
$$

$$
u_{\mathrm{eq}}(x)=\frac{\alpha\left(\omega_{1} x_{3}+k \omega_{2} x_{4}-e\right)-\beta\left(k \omega_{1} x_{3}+\omega_{2} x_{4}-k e\right)+\left(-\gamma \omega_{1} x_{1}+\delta \omega_{2} x_{2}+\delta \omega_{R} x_{4}\right)\left(1-k^{2}\right)}{\alpha\left(\omega_{1}-k \omega_{21}\right) x_{3}+\beta\left(\omega_{21}-k \omega_{1}\right) x_{3}-\gamma\left(1-k^{2}\right)\left(\omega_{1} x_{1}+\omega_{21} x_{2}\right)}
$$

$$
x_{4}^{*}=\frac{-\left(\delta-\frac{\beta \omega_{R}}{\omega_{2}}-\frac{\gamma \omega_{2}}{\omega_{21}}\right) \pm \sqrt{\left(\delta-\frac{\beta \omega_{R}}{\omega_{2}}-\frac{\gamma \omega_{2}}{\omega_{21}}\right)^{2}-\frac{4 \alpha \omega_{R}}{e}\left(\frac{\gamma e}{\omega_{1}}-\theta\right)}}{\frac{2 \alpha \omega_{R}}{e}} \quad \text { if } \alpha \neq 0
$$


Moreover, the expression of the equivalent control in the equilibrium point will be given by

$$
u_{\mathrm{eq}}^{*}=\frac{1}{1-\frac{\omega_{21} e}{\omega_{1} \omega_{2}} \frac{1}{x_{4}^{*}}} .
$$

From (18) and (30), we obtain

$$
0<-\frac{\omega_{21} e}{\omega_{1} \omega_{2}} \frac{1}{x_{4}^{*}} \lessgtr 1
$$

which in terms of the physical variables of the converter can be expressed as

$$
0<-\frac{V_{g}}{v_{C o}^{*}} \gtrless 1 \text {. }
$$

Inequality (32) describes the sign inversion in the output voltage of the Cuk converter and the possibility of stepdown or step-up characteristics. It also establishes under which conditions an equilibrium point belongs to the sliding domain.

\section{SLIDING SURFACES}

\section{A. Sliding Surfaces of the Form $x_{i}=\theta$}

There are three sliding surfaces of the form $x_{i}=\theta$ which satisfy the transversality condition. Namely,

$$
\begin{aligned}
& x_{1}=\theta \\
& x_{2}=\theta \\
& x_{3}=\theta .
\end{aligned}
$$

In the first case, the ideal sliding dynamics can be described by the following set of equations:

$$
\begin{aligned}
\dot{x}_{2}=- & \frac{k e}{1-k^{2}}+\frac{k \omega_{1} x_{3}}{1-k^{2}}+\frac{\omega_{2} x_{4}}{1-k^{2}}+\frac{\omega_{21}-k \omega_{1}}{1-k^{2}} \\
& \cdot \frac{\omega_{1} x_{3}+k \omega_{2} x_{4}-e}{\omega_{1}-k \omega_{21}} \\
\dot{x}_{3}= & \omega_{1} x_{1}-\left(\omega_{1} x_{1}+\omega_{21} x_{2}\right) \frac{\omega_{1} x_{3}+k \omega_{2} x_{4}-e}{\left(\omega_{1}-k \omega_{21}\right) x_{3}} \\
\dot{x}_{4}= & -\omega_{2} x_{2}-\omega_{R} x_{4} .
\end{aligned}
$$

The asymptotic behavior of (36)-(38) can be represented by a stable equilibrium point whose coordinates are the following:

$$
\begin{aligned}
& x_{1}^{*}=\theta, x_{2}^{*}=\frac{\sqrt{\omega_{R} e \theta}}{\omega_{2}}, \\
& x_{3}^{*}=\frac{e}{\omega_{1}}+\frac{\omega_{2}}{\omega_{21}} \sqrt{\frac{e \theta}{\omega_{R}}}, x_{4}^{*}=-\sqrt{\frac{e \theta}{\omega_{R}}} .
\end{aligned}
$$

The local stability conditions for this equilibrium point can be summarized as follows:

1) for $k \geq 0$, the system will be stable if $k \omega_{21}-\omega_{1}<0$; and

2) for $k<0$, the system is stable for values of $x_{4}^{*}$ near zero, becoming unstable for large values of $\left|x_{4}^{*}\right|$.

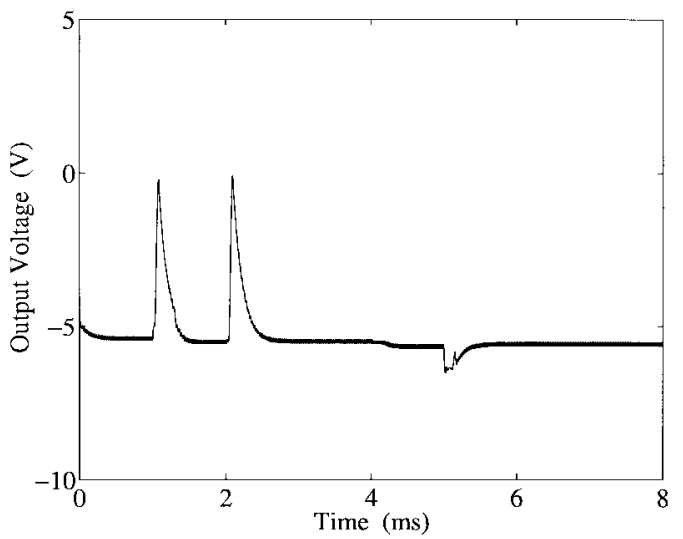

Fig. 2. Output voltage response to input and load variations (Figs. 3 and 4 ) when the sliding mode uses the surface $2 \cdot 10^{-5} d v_{C o} / d t+v_{C o}+5=0$.

The corresponding control law will be expressed as follows:

$$
\begin{array}{ll}
u=1 & \text { if } x_{1}-\theta<0 \\
u=0 & \text { if } x_{1}-\theta>0 .
\end{array}
$$

In the second case, the discontinuity surface $x_{2}=\theta$ yields an ideal sliding dynamics containing a stable equilibrium point, provided that the following condition is satisfied:

$$
\frac{k e}{\omega_{2}}<x_{4}^{*}<0
$$

or, equivalently,

$$
k V_{g} \sqrt{\frac{L_{2}}{L_{1}}}<v_{C o}^{*}<0
$$

which implies that $k$ must be negative.

On the other hand, if we consider the sliding surface $x_{3}=\theta$, we obtain an unstable equilibrium point.

Although the sliding surface $x_{4}=\theta$ does not satisfy the transversality condition, we can take the following surface:

$$
\frac{d x_{4}}{d t}+\delta x_{4}=\theta
$$

which satisfies the transversality condition and results in the equivalent control [see (45), shown at the bottom of the page].

The corresponding ideal dynamics have a stable equilibrium point provided that condition (42) is accomplished and $\delta$ is positive.

In Fig. 2, a SIMPLIS simulation [25]-[27] shows the output voltage behavior when the input voltage and the load resistance exhibit simultaneously the pulsating characteristics depicted in Figs. 3 and 4, respectively. The converter parameters are $L_{1}=L_{2}=1 \mathrm{mH}, k=-0.9, C_{1}=1 \mu \mathrm{F}$, and $C_{o}=20 \mu \mathrm{F}$.

$$
u_{\mathrm{eq}}=\frac{k \omega_{2} \omega_{1} x_{3}+\omega_{2}^{2} x_{4}-k \omega_{2} e+\left(1-k^{2}\right)\left(\delta-\omega_{R}\right)\left(\omega_{2} x_{2}+\omega_{R} x_{4}\right)}{\left(k \omega_{1}-\omega_{21}\right) \omega_{2} x_{3}}
$$




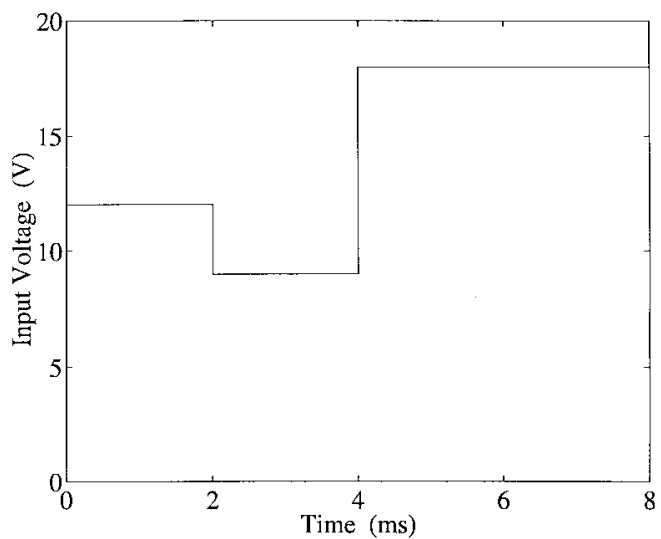

Fig. 3. Input voltage characteristics.

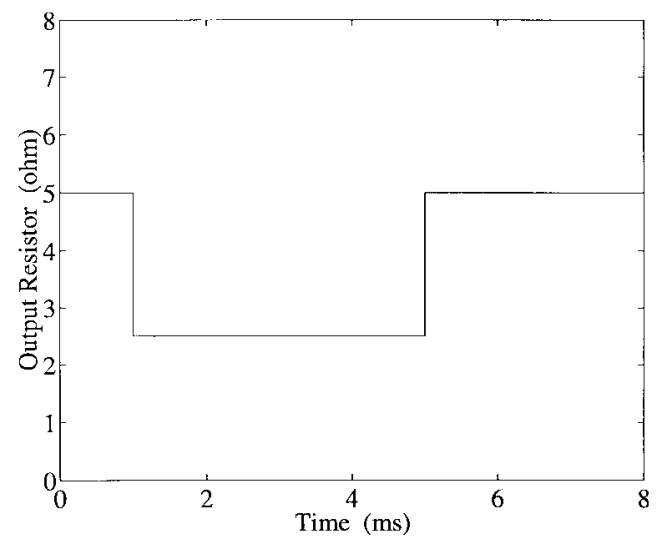

Fig. 4. Pulsating characteristics of the load.

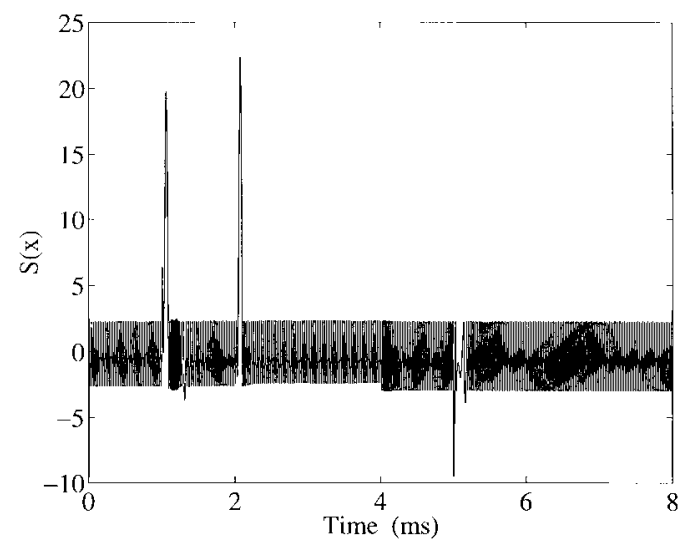

Fig. 5. Sliding surface behavior corresponding to Fig. 2.

The comparator hysteresis has been adjusted to provide a switching frequency around $50 \mathrm{kHz}$. The sliding surface is similar to a linear compensating network of PD type. The analogy suggests that the inclusion of an integral term in the surface would eliminate the steady-state error [33].

Fig. 5 illustrates the time-domain behavior of the sliding surface. There is loss of sliding regime at $t=2 \mathrm{~ms}$ corresponding to the first discontinuity of the input voltage and at instants corresponding to discontinuities in the output load. The loss of sliding regime is reflected at the same instants in the transientstate of the output voltage depicted in Fig. 2. However, if the input voltage has soft transitions between levels as shown in Fig. 6, there is a significant improvement of the transient

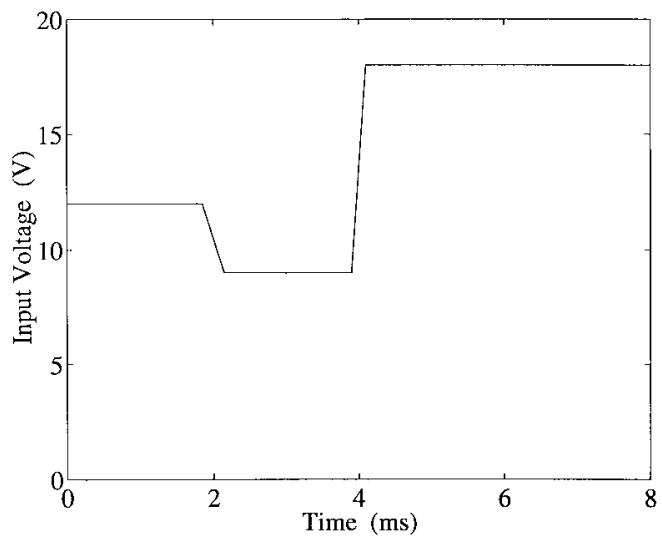

Fig. 6. Input voltage with soft transitions.

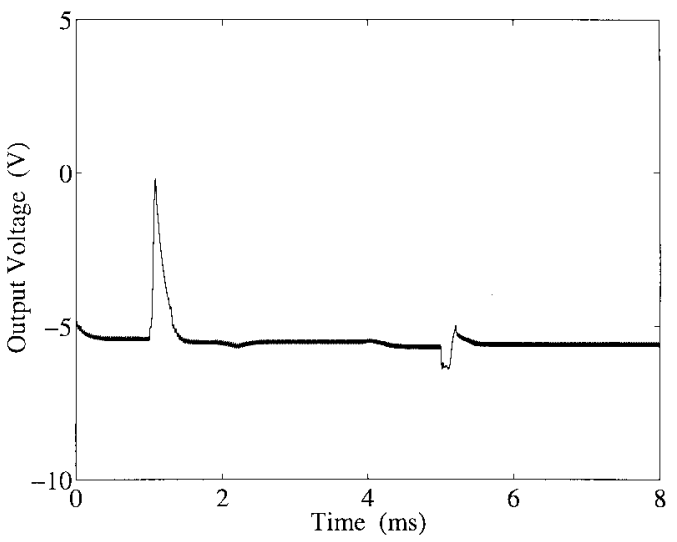

Fig. 7. Output voltage response to input and load variations (Figs. 4 and 6) when the sliding mode uses the same surface as in Fig. 2.

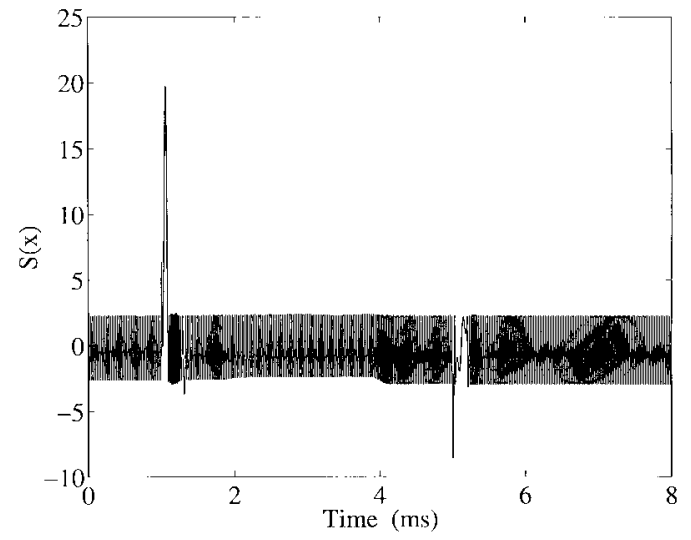

Fig. 8. Sliding surface behavior corresponding to Fig. 7.

behavior of the output voltage and no loss of sliding regime as depicted in Figs. 7 and 8, respectively.

\section{B. Discontinuity Surfaces for Ideal Line Regulation}

We have to choose those surfaces leading to sliding dynamics with equilibrium points in which the coordinate of the output variable $x_{4}^{*}$ does not depend on $e$.

From (28) and (29), we conclude that the surfaces resulting in ideal line regulation will be of the form

$$
\beta x_{2}+\delta x_{4}=\theta
$$




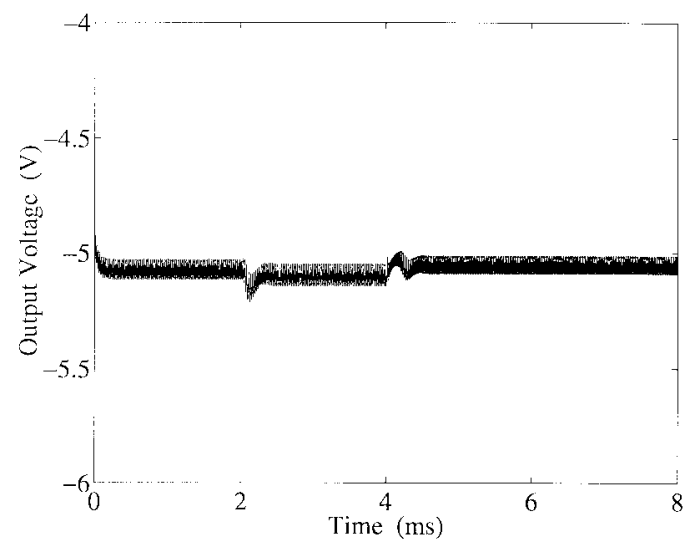

Fig. 9. Output voltage response to the input variations depicted in Fig. 3 when the sliding control uses the surface $i_{L 2}-0.2 v_{C o}-2=0$.

The ideal sliding dynamics will be described as

$$
\begin{aligned}
\dot{x}_{1}= & \frac{e-\omega_{1} x_{3}-k \omega_{2} x_{4}}{1-k^{2}}+\frac{\omega_{1}-k \omega_{21}}{1-k^{2}} \\
& \cdot \frac{-\beta\left(k \omega_{1} x_{3}+\omega_{2} x_{4}-k e\right)+\delta\left(\omega_{2} x_{2}+\omega_{R} x_{4}\right)\left(1-k^{2}\right)}{\beta\left(\omega_{21}-k \omega_{1}\right)}
\end{aligned}
$$

$\dot{x}_{2}=\frac{\delta}{\beta}\left(\omega_{2} x_{2}+\omega_{R} x_{4}\right)$

$$
\begin{aligned}
\dot{x}_{3}= & \omega_{1} x_{1}-\left(\omega_{1} x_{1}+\omega_{21} x_{2}\right) \\
& \cdot \frac{-\beta\left(k \omega_{1} x_{3}+\omega_{2} x_{4}-k e\right)+\delta\left(\omega_{2} x_{2}+\omega_{R} x_{4}\right)\left(1-k^{2}\right)}{\beta\left(\omega_{21}-k \omega_{1}\right) x_{3}}
\end{aligned}
$$

$\dot{x}_{4}=-\omega_{2}\left(\frac{\theta}{\beta}-\frac{\delta}{\beta} x_{4}\right)-\omega_{R} x_{4}$.

Since (50) does not depend on $x_{2}$ nor on $x_{3}, x_{4}(t)$ will be given by

$$
x_{4}(t)=K_{1} e^{-\left(\omega_{R}-\omega_{2} \delta / \beta\right) t}-\frac{\omega_{2} \frac{\theta}{\beta}}{\omega_{R}-\frac{\omega_{2} \delta}{\beta}}
$$

which exhibits an asymptotically stable equilibrium point in $x_{4}^{*}=-\left[\theta \omega_{2} /\left(\beta \omega_{R}-\delta \omega_{2}\right)\right]$ provided that $\omega_{R}>\omega_{2} \delta / \beta$.

Similarly, from (48) $x_{2}(t)$ will be expressed as

$$
x_{2}(t)=K_{2} e^{-\left(\omega_{R}-\omega_{2} \delta / \beta\right) t}+\frac{\theta \omega_{R}}{\beta \omega_{R}-\delta \omega_{2}}
$$

which reaches the stable equilibrium point $x_{2}^{*}=\theta \omega_{R} /\left(\beta \omega_{R}-\right.$ $\left.\delta \omega_{2}\right)$.

Equations (47) and (49), in turn, result in an equilibrium point

$$
\left(\frac{\omega_{R} x_{4}^{* 2}}{e}, \frac{e}{\omega_{1}}-\frac{\omega_{2}}{\omega_{21}} x_{4}^{*}\right)
$$

which is stable if (42) is satisfied.

In Fig. 9, a SIMPLIS simulation illustrates the insensitivity in steady state of the output voltage-to-input voltage variations. The converter parameters are $L_{1}=L_{2}=1 \mathrm{mH}, k=-0.9$, $C_{1}=1 \mu \mathrm{F}, C_{o}=20 \mu \mathrm{F}, R_{o}=5 \Omega$, and the input voltage has the time-varying behavior depicted in Fig. 3.

\section{Ideal Load Regulation}

Considering the surface $\alpha x_{1}+\beta x_{2}=0$, the following ideal dynamics are obtained:

$$
\begin{aligned}
\dot{x}_{2}= & \frac{-k e+k \omega_{1} x_{3}+\omega_{2} x_{4}}{1-k^{2}}+\frac{\omega_{21}-k \omega_{1}}{1-k^{2}} \\
& \cdot \frac{\omega_{1} x_{3}+k \omega_{2} x_{4}-e-\beta\left(k \omega_{1} x_{3}+\omega_{2} x_{4}-k e\right)}{\left(\omega_{1}-k \omega_{21}\right)+\beta\left(\omega_{21}-k \omega_{1}\right)} \\
\dot{x}_{3}= & -\beta \omega_{1} x_{2}+\left(\beta \omega_{1}-\omega_{21}\right) \frac{x_{2}}{x_{3}} \\
& \cdot \frac{\omega_{1} x_{3}+k \omega_{2} x_{4}-e-\beta\left(k \omega_{1} x_{3}+\omega_{2} x_{4}-k e\right)}{\left(\omega_{1}-k \omega_{21}\right)+\beta\left(\omega_{21}-k \omega_{1}\right)} \\
\dot{x}_{4}= & -\omega_{2} x_{2}-\omega_{R} x_{4}
\end{aligned}
$$

where we have considered $\alpha=1$ without loss of generality.

The coordinates of the equilibrium point are

$$
\begin{aligned}
& x_{1}^{*}=\frac{\beta \omega_{R}}{\omega_{2}} x_{4}^{*}, x_{2}^{*}=-\frac{\omega_{R}}{\omega_{2}} x_{4}^{*}, \\
& x_{3}^{*}=\frac{e}{\omega_{1}}-\frac{\omega_{2}}{\omega_{21}} x_{4}^{*}, x_{4}^{*}=\frac{\beta e}{\omega_{2}} .
\end{aligned}
$$

From (32) and (56) we derive

$$
\beta<0 .
$$

Applying the Routh's criterion to the characteristic polynomial of the linearized equations around the equilibrium point, it can be shown that the system will be asymptotically stable if $\beta$ satisfies the inequality

$$
-\frac{\sqrt{L_{2}}-k \sqrt{L_{1}}}{\sqrt{L_{1}}-k \sqrt{L_{2}}}<\beta<0 \quad 0 \leq k<1 .
$$

Considering $\alpha \neq 1$, the stability condition becomes

$$
-\frac{\sqrt{L_{2}}-k \sqrt{L_{1}}}{\sqrt{L_{1}}-k \sqrt{L_{2}}}<\frac{\beta}{\alpha}<0 .
$$

Equivalently,

$$
-\frac{L_{2}-M}{L_{1}-M}<\frac{m_{2}}{m_{1}}<0 .
$$

Similarly, we can express the surface for ideal load regulation in terms of the physical variables

$$
m_{1} i_{L 1}+m_{2} i_{L 2}=0 .
$$

The coordinate of the output voltage will be given by

$$
v_{C o}^{*}=\frac{m_{2}}{m_{1}} V_{g}
$$

which is independent of the output load $R_{o}$.

The sliding domain for the output voltage will be expressed as

$$
\begin{array}{ll}
\left|v_{C o}^{*}\right|<V_{g} & \text { if }\left|\frac{m_{2}}{m_{1}}\right|<1 \quad \text { (step-down behavior) } \\
\left|v_{C o}^{*}\right|>V_{g} & \text { if }\left|\frac{m_{2}}{m_{1}}\right|>1 \quad \text { (step-up behavior). }
\end{array}
$$

From (61) and (62), we obtain

$$
V_{g} i_{L 1}^{*}=-v_{C o}^{*} i_{L 1}^{*}
$$




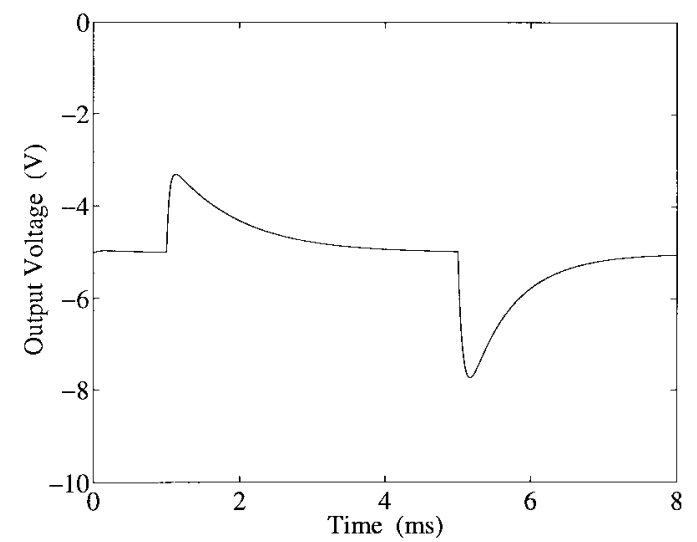

Fig. 10. Output voltage response to a pulsating load shown in Fig. 4 when the sliding surface is $i_{L 1}-0.42 i_{L 2}=0$.

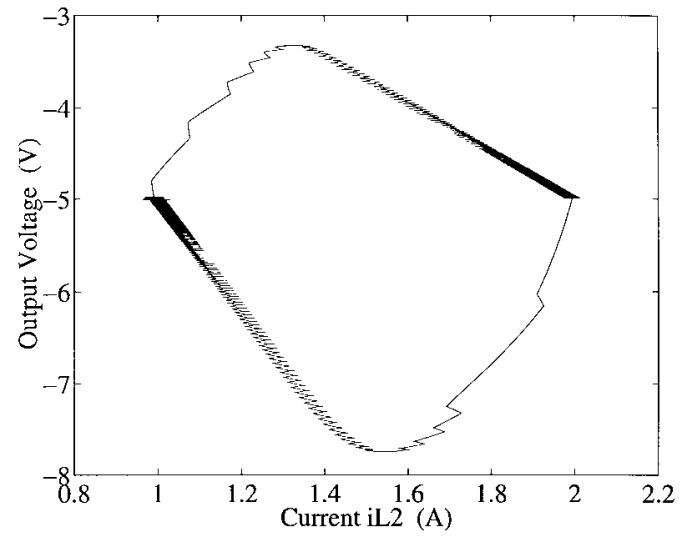

Fig. 11. Trajectories in the plane $i_{L 2}-v_{C o}$ when the load has the pulsating behavior of Fig. 4.

or, equivalently,

$$
P \mathrm{dc}_{\mathrm{in}}=P \mathrm{dc}_{O}
$$

where $P \mathrm{dc}_{\text {in }}$ and $P \mathrm{dc}_{o}$ are input and output power, respectively.

As no internal losses are considered, equality (66) could be expected which verifies the validity of the former results.

By comparing (61) to the general sliding surface (10), we conclude that there are no external references in the feedback loop and, therefore, the circuit will behave as a self-oscillating regulator. The corresponding control law is given by

$$
\begin{array}{ll}
u=0 & \text { if } m_{1} i_{L 1}+m_{2} i_{L 2}>0 \\
u=1 & \text { if } m_{1} i_{L 1}+m_{2} i_{L 2}<0 .
\end{array}
$$

As an example, for the set of parameters $L_{1}=L_{2}=1$ $\mathrm{mH}, k=0.9, C_{1}=C_{o}=20 \mu \mathrm{F}$, and $V_{g}=12 \mathrm{~V}$, the converter provides $-5 \mathrm{~V}$ in the output, and exhibits the transient behavior depicted in the SIMPLIS simulation of Fig. 10 when the load has the pulsating characteristics represented in Fig. 4. The converter sliding behavior in the plane $i_{L 2}-v_{C o}$ is illustrated in Fig. 11. As shown in Figs. 10 and 11, the output voltage recovers its steady-state value of $-5 \mathrm{~V}$ after the transient states that follow the load changes.

\section{Sliding Surfaces for Hysteretic Current Control}

Hysteretic current control has been proposed in the coupledinductor Ćuk converter [28] as an alternative to the constant frequency peak current control, in order to avoid the intrinsic instability for duty cycles larger than 0.5 , and the output jittering caused by the magnetic coupling. The hysteretic current control implemented in [28] can also be described in terms of the sliding-mode approach developed in this paper. Assuming $m_{1}=m_{2}=1$ and $m_{3}=m_{4}=0$ in the general sliding surface (10), we obtain

$$
S(x)=\left(i_{L 1}+i_{L 2}\right)-\left(i_{L 1}^{*}+i_{L 2}^{*}\right) .
$$

In terms of the normalized variables, the ideal sliding dynamics will be given by

$$
\begin{aligned}
\dot{x}_{2}= & \frac{k \omega_{1} x_{3}+\omega_{2} x_{4}-k e}{1-k^{2}}+\frac{\omega_{21}-k \omega_{1}}{1-k^{2}} \\
& \cdot \frac{\alpha\left(\omega_{1} x_{3}+k \omega_{2} x_{4}-e\right)-\beta\left(k \omega_{1} x_{3}+\omega_{2} x_{4}-k e\right)}{\alpha\left(\omega_{1}-k \omega_{21}\right)+\beta\left(\omega_{21}-k \omega_{1}\right)} \\
\dot{x}_{3}= & \omega_{1} \frac{\theta-\beta x_{2}}{\alpha}+\left(-\omega_{1} \frac{\theta-\beta x_{2}}{\alpha}-\omega_{21} x_{2}\right) \\
& \cdot \frac{\alpha\left(\omega_{1} x_{3}+k \omega_{2} x_{4}-e\right)-\beta\left(k \omega_{1} x_{3}+\omega_{2} x_{4}-k e\right)}{\alpha\left(\omega_{1}-k \omega_{21}\right)+\beta\left(\omega_{21}-k \omega_{1}\right)} \\
\dot{x}_{4}= & -\omega_{2} x_{2}-\omega_{R} x_{4} .
\end{aligned}
$$

Equations (70)-(72) have an equilibrium point in

$$
\begin{aligned}
& x_{1}^{*}=\frac{\theta-\beta x_{4}^{*}}{\alpha}, x_{2}^{*}=-\frac{\omega_{R}}{\omega_{2}} x_{4}^{*}, x_{3}^{*}=\frac{e}{\omega_{1}}-\frac{\omega_{2}}{\omega_{21}} x_{4}^{*}, \\
& x_{4}^{*}=\frac{\frac{\beta \omega_{R}}{\omega_{2}}-\sqrt{\left(\frac{\beta \omega_{R}}{\omega_{2}}\right)^{2}-\frac{4 \theta \alpha \omega_{R}}{e}}}{\frac{2 \alpha \omega_{R}}{e}} .
\end{aligned}
$$

The analysis of the linearized sliding dynamics around the equilibrium point shows that we can always find a subinterval $x_{4 b}<x_{4}^{*}<0$ where the system is stable, $x_{4 b}$ being finite and depending on the converter parameters.

The control law in this case is given by

$$
\begin{array}{ll}
u=0 & \text { if } i_{S}>i_{L 1}^{*}+i_{L 2}^{*} \\
u=1 & \text { if } i_{S}<i_{L 1}^{*}+i_{L 2}^{*}
\end{array}
$$

where $i_{S}$ represents the switch current defined as

$$
i_{S}=i_{1}+i_{2} \text {. }
$$

The implementation of this control law leads straightforwardly to the hysteretic current-controlled Ćuk converter presented in [28].

\section{CONCLUSIONS}

Analytic solutions have been derived for the dynamic behavior of a bidirectional coupled-inductor Ćuk converter operating in sliding mode. The method of the equivalent control has been successfully applied to describe the converter ideal sliding dynamics under different discontinuity surfaces. 
Insensitivity in steady state to output-voltage variations can be obtained for certain values of the output voltage if the discontinuity surface is a linear combination of output voltage and output current, and the magnetic coupling between inductors is negative.

It has been demonstrated that the sensitivity of the output voltage to load variations can be reduced to zero in steady state if the regulator operates in a self-oscillating mode and the sliding surface is a linear combination with opposite signs of input and output currents. The inclusion of an external reference in the previous surface and the assumption of equal coefficients with equal signs show that the hysteretic current control can be described as a particular case of the sliding mode control.

Another important conclusion is that the converter can be controlled like a buck switching cell using a linear conbination of the output voltage error and its derivative and minimum size of energy transfer capacitor.

The general sliding surface analyzed in this paper can also be applied in the control of other four-order structures [29]-[32]. Further research contemplates the implementation of the sliding controller by means of analog ASIC technology.

\section{REFERENCES}

[1] S. Ćuk, "Switching dc-to-dc converter with zero input or output current ripple," in Proc. IEEE Industry Appl. Soc. Annu. Meet., Toronto, Ont., Canada, 1978, pp. 1131-1146.

[2] A. Cocconi and S. Ćuk, "Design of a $2 \mathrm{~kW}, 100 \mathrm{kHz}$, switching regulator for space shuttle," in Proc. PCI, Sept. 1982, pp. 72-85.

[3] S. Singer, "Loss-free gyrator realization," IEEE Trans. Circuits Syst., vol. 35, pp. 26-34, Jan. 1988.

[4] R. D. Middlebrook and S. Ćuk, "Advances in switched-mode power conversion," in Modeling, Analysis and Measurement. Pasadena, CA: Teslaco, 1981, vol. I.

[5] S. Cuk and R. D. Middlebrook, "Advances in switched-mode power conversion" (Parts I and II), IEEE Trans. Ind. Electron., vol. IE-30, pp. 10-29, Feb. 1983.

[6] F. Ueno, T. Inone, F. Tsuruta, and I. Oota, "Steady state and dynamic analyses of Ćuk converter in the continuous and discontinuous mode," in Proc. ISCAS'85, pp. 1007-1010.

[7] L. Martínez, A. Poveda, and J. M. Miguel, "Modeling and analysis of the Ćuk convertor using the discrete impulse response method," Proc. Inst. Elect. Eng., vol. 133, pt. G, no. 2, pp. 77-83, Apr. 1986.

[8] A. Pietkiewicz and D. Tollik, "Modeling and analysis of the currentmode controlled Cuk converter with coupled inductors," in PESC' 88 Rec., pp. 398-408.

[9] S. Cuk, "Discontinuous inductor current mode in the optimum topology switching dc-to-dc converter," in PESC'77 Rec., pp. 105-123.

[10] S. Ćuk and R. Erickson, "A conceptually new high-frequency switchedmode amplifier technique eliminates current ripple," in Proc. 5th Nat. Solid-State Power Conversion Conf., San Francisco, 1978, pp. G.3.1-G.3.22.

[11] V. I. Utkin, Sliding Modes and Their Application in Variable Structure Systems. Moscow, Russia: Mir, 1974.

[12] U. Itkis, Control Systems of Variable Structure. New York: Wiley, 1976.

[13] F. Bilalovic, O. Music, and A. Sabanovic, "Buck converter operating in the sliding mode," in Proc. PCI'83, pp. 331-340.

[14] R. Venkataraman, "Sliding mode control of power converters," Ph.D. dissertation, Calif. Inst. Technol., Pasadena, 1986.

[15] R. Venkataraman, A. Sabanovic, and S. Ćuk, "Sliding mode control of dc-to-dc converters," in Proc. IECON'85, pp. 251-258.

[16] S. Sanders, G. C. Verghese, and D. F. Cameron, "Nonlinear control laws for switching power converters," in Proc. 25th Conf. Decision Contr., 1986, pp. 46-53.

[17] H. Sira-Ramirez, "Sliding motions in bilinear switched networks," IEEE Trans. Circuits Syst., vol. CAS-34, pp. 919-933, 1987.

[18] S.-P. Huang, H.-Q. Xu, and Y.-F. Liu, "Sliding-mode controlled Ćuk switching regulator with fast response and first-order dynamic characteristic," in PESC'89 Rec., pp. 124-129.
[19] E. Fossas, L. Martinez, and J. Ordinas, "Sliding mode control reduces audiosusceptibility and load perturbations in the Cuk converter," IEEE Trans. Circuits Syst., vol. CAS-39, pp. 847-849, 1992.

[20] L. Malesani, L. Rosetto, G. Spiazzi, and P. Tenti, "Performance optimization of Ćuk converters by sliding-mode control," IEEE Trans. Power Electron., vol. 10, pp. 302-309, 1995.

[21] M. Oppenheimer, I. Husain, M. Ebuluk, and J. A. De Abreu-Garcia, "Sliding mode control of the Ćuk converter," in PESC'96 Rec., pp. $1519-1526$.

[22] J. Majó, L. Martínez, A. Poveda, L. García de Vicuña, F. Guinjoan, A. F. Sánchez, M. Valentin, and J. C. Marpinard, "Large-signal feedback control of a bidirectional coupled-inductor Cuk converter," IEEE Trans. Ind. Electron., vol. 39, pp. 429-436, Oct. 1992.

[23] J. Majó, L. Martínez, A. Poveda, I. García de Vicuña, F. Guinjoan, A. F Sánchez, J. C. Marpinard, and M. Valentin, "Nonlinear continuous time control of bidirectional coupled-inductor Ćuk converter," IEICE Trans. Commun., vol. E-75B, no. 11, pp. 1134-1141, Nov. 1992.

[24] I. M. H. Bábáa, T. G. Wilson, and Y. Tu, "Analytic solutions of limit cycles in a feedback-regulated converter system with hysteresis," IEEE Trans. Automat. Contr., vol. AC-13, pp. 524-531, Oct. 1968.

[25] SIMPLIS, Simulation Software for Switching Power Supply Design, Technical Reference Guide. Los Altos, CA: Power Design Tools, Inc.

[26] R. C. Wong, "Control of energy-storage dc-to-dc converters: The largesignal time domain approach,” Ph.D. dissertation, Duke Univ., Durham, NC, 1984.

[27] R. C. Wong, H. A. Owen, and T. G. Wilson, "An efficient algorithm for the time-domain simulation of regulated energy-storage dc-to-dc converters," IEEE Trans. Power Electron., vol. PE-2, pp. 154-168, Apr. 1987.

[28] S.-C. Wong and Y.-S. Lee, "SPICE modeling and simulation of hysteretic current-controlled Cuk converter," IEEE Trans. Power Electron. vol. 8, pp. 850-857, Oct. 1993

[29] F. Dominguez, E. Fossas, R. Giral, and L. Martínez, "Boost converter with output filter. A sliding approach," in Proc. 37th Midwest Symp. Circuits Syst., Lafayette, LA, Aug. 3-5, 1994, pp. 1265-1268.

[30] F. Dominguez, E. Fossas, and L. Martínez, "Stability analysis of a buck converter with input filter via sliding-mode approach," in Proc. IECON'94, 20th Annu. Int. Conf. Ind. Electron., Contr., Instrumentat., and Automat., Bologna, Italy, Sept. 5-9, 1994, pp. 1438-1442.

[31] J. Hernanz, L. Martínez, A. Poveda, and E. Fossas, "Analysis of slidingmode controlled SEPIC converter," in Proc. IPEC'95, Power Electron. Conf., Yokohama, Japan, Apr. 3-7, 1995, pp. 422-427.

[32] J. Hernanz, L. Martínez, R. Giral, A. Poveda, and E. Fossas, "Analysis of a sliding-mode controlled dual SEPIC converter," in Proc. 1995 European Conf. Circuit Theory Design, Istanbul, Turkey, Aug. 27-31, 1995 , pp. $1165-1168$.

[33] R. Giral, L. Martínez, J. Hernanz, J. Calvente, F. Guinjoan, A. Poveda, and R. Leyva, "Compensating networks for sliding-mode control," in Proc. ISCAS'95, IEEE Int. Symp. Circuits Syst., Seattle, WA, Apr. 29-May 3, 1995, pp. 2055-2058.

[34] D. Redfern, Maple V, Release 4, The Maple Handbook. New York: Springer-Verlag, 1996. (Maple is a registered trademark of Waterloo Maple, Inc.)

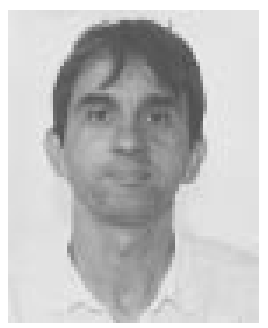

Luis Martínez-Salamero (M'79-SM'86) graduated as an Ingeniero de Telecomunicación in 1978, and received the doctor's degree in 1984, both from the Universidad Politécnica de Cataluña, Spain.

From 1978 to 1992, he taught circuit theory, analog electronics, and power processing at the Escuela Técnica Superior de Ingenieros de Telecomunicación de Barcelona. During the academic year 1992-1993, he was a Visiting Professor at the Center for Solid State Power Conditioning and Control, Department of Electrical Engineering, Duke University, Durham, NC. He is currently a Professor at the Departamento de Ingeniería Electrónica, Escuela Técnica Superior de Ingeniería, Universitat Rovira i Virgili, tarragona, Spain. His research interests are in the field of structure and control of power conditioning systems. 


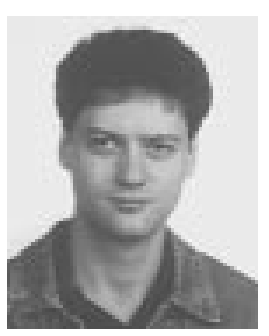

Javier Calvente graduated as an Ingeniero de Telecomunicación in 1994 from the Universidad Politécnica de Cataluña, Spain.

$\mathrm{He}$ is currently an Assistant Professor at the Departamento de Ingeniería Electrónica, Escuela Técnica Superior de Ingeniería, Universitat Rovira i Virgili, Tarragona, Spain, where he is working toward the Ph.D. degree in the field of nonlinear control of power converters.

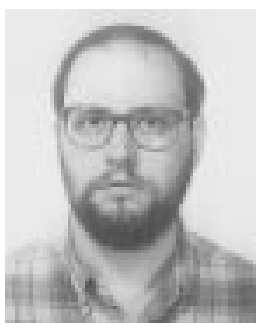

Roberto Giral graduated as an Ingeniero Técnico de Telecomunicación and Ingeniero de Telecomunicación in 1990 and in 1994, respectively, from the Universidad Politécnica de Cataluña.

$\mathrm{He}$ is currently an Assistant Professor at the Departamento de Ingeniería Electrónica, Escuela Técnica Superior de Ingeniería, Universitat Rovira i Virgili, Tarragona, Spain, where he is working toward the Ph.D. degree in the field of power converters.

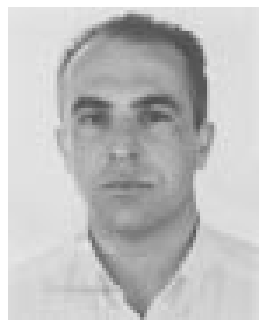

Alberto Poveda received the Ingeniero de Telecomunicación and Doctor Ingeniero degrees from the Universidad Politécnica de Cataluña, Spain, in 1978 and 1988 , respectively.

$\mathrm{He}$ is presently an Associate Professor at the Escuela Técnica Superior de Ingenieros de Telecomunicación de Barcelona, Spain, where he teaches analog circuits and power electronics. His research interests include analog circuits design and power processing modeling, control, and simulation.

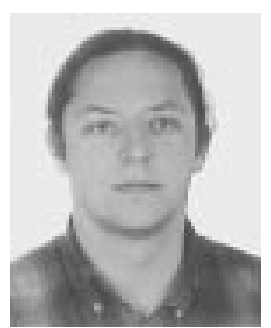

Enric Fossas received the Graduate and Ph.D. degrees in mathematics from Barcelona University, Spain, in 1981 and 1986, respectively.

Since 1981, he has taught mathematics at the Universitat de Barcelona and at the Universitat Politécnica de Catalunya where he is employed as an Associate Professor. His research interests are in the field of system theory, control, and differential geometry. 